\title{
Fatores associados ao envolvimento de gestantes com álcool e outras drogas
}

Factors associated with the of pregnant women involved with alcohol and other drugs

Factores asociados a la participación de gestantes con alcohol y otras drogas

Priscilla Nunes Porto ${ }^{1 *}$, Daine Ferreira Brazil do Nascimento ${ }^{1}$, Georgiane Silva Mota ${ }^{1}$, Mayara Novais Pereira ${ }^{1}$, Dejeane de Oliveira Silva ${ }^{2}$, Carle Alberto Porcino ${ }^{1}$, Jeane Freitas de Oliveira ${ }^{1}$.

\section{RESUMO}

Objetivo: estimar a prevalência e verificar fatores associados ao envolvimento de gestantes com substâncias psicoativas. Métodos: estudo transversal com 268 gestantes cadastradas no pré-natal de uma maternidade pública do município de Salvador - BA. Os dados foram processados e analisados no software estatístico SPSS 20 e apresentados mediante números absolutos e índices percentuais. Para a análise bivariada utilizouse o Teste Exato de Fisher e odds ratio com intervalo de confiança de $95 \%$. Resultados: a prevalência do envolvimento de gestantes com álcool e outras drogas foi de $98,1 \%$. Observou-se associação estatística entre o consumo de substâncias psicoativas pela gestante e raça $(0,020)$, escolaridade $(0,017)$, religião $(0,010)$ e condição de moradia $(0,014)$. Conclusão: o envolvimento de gestantes com as drogas se constitui como elemento que potencializa as vulnerabilidades. A identificação e a associação do envolvimento com fatores sociodemográficos demandam aos profissionais de saúde o desenvolvimento de estratégias de intervenção primando pelas singularidades e especificidades dessas mulheres para minimizar as vulnerabilidades vivenciadas por elas.

Palavras-chave: Gestante, Drogas, Estudo sobre vulnerabilidade, Enfermagem.

\begin{abstract}
Objective: to estimate the prevalence and verify factors associated with the involvement of pregnant women with psychoactive substances. Method: a cross-sectional study with 268 pregnant women enrolled in prenatal care at a public maternity hospital in the city of Salvador - Bahia. The data were processed and analyzed in SPSS 20 statistical software and presented using absolute numbers and percentage indices. For the bivariate analysis Fisher's Exact Test and odds ratio with 95\% confidence interval were used. Results: the prevalence of involvement of pregnant women with alcohol and other drugs was $98.1 \%$. Statistical association between psychoactive substances use by pregnant women and race $(0.020)$, schooling $(0.017)$, religion $(0.010)$ and housing condition (0.014) were observed. Conclusion: the involvement of pregnant women with drugs constitutes an element that enhances vulnerabilities. The identification and association of involvement with sociodemographic factors demand from health professionals the development of intervention strategies emphasizing the singularities and specificities of these women to minimize the vulnerabilities experienced by them.
\end{abstract}

Key words: Pregnant women, Drugs, Vulnerability study, Nursing.

1Universidade Federal da Bahia (UFBA), Salvador - BA. *E-mail: priscillaporto@outlook.com

${ }^{2}$ Universidade Estadual de Santa Cruz (UESC), llhéus - Ba

Pesquisa financiada pelo CNPQ

SUBMETIDO EM: 4/2019

ACEITO EM: 5/2019

PUBLICADO EM: 7/2019

REAS/EJCH |Vol. 11(12) | e795 | DOI: https://doi.org/10.25248/reas.e795.2019 Página 1 de 8 


\section{RESUMEN}

Objetivo: estimar la prevalencia y verificar los factores asociados con la participación de mujeres embarazadas con sustancias psicoactivas. Método: un estudio transversal con 268 mujeres embarazadas inscritas en cuidados prenatales en un hospital público de maternidad en la ciudad de Salvador - Bahía. Los datos se procesaron y analizaron en el software estadístico SPSS 20 y se presentaron utilizando números absolutos e índices porcentuales. Para el análisis bivariado se utilizaron la prueba exacto de Fisher y el odds ratio con un intervalo de confianza del 95\%. Resultados: la prevalencia de participación de mujeres embarazadas con alcohol y otras drogas fue del 98,1\%. Se observó asociación estadística entre el uso de sustancias psicoactivas por mujeres embarazadas y la raza (0.020), la escolarización $(0.017)$, la religión (0.010) y la condición de la vivienda (0.014). Conclusión: la participación de mujeres embarazadas con drogas constituye un elemento que mejora las vulnerabilidades. La identificación y asociación de la participación con factores sociodemográficos exigen a los profesionales de la salud el desarrollo de estrategias de intervención que enfaticen las singularidades y especificidades de estas mujeres para minimizar las vulnerabilidades que experimentan.

Palabras-clave: Mujeres embarazadas, Drogas, Estudio de vulnerabilidade, Enfermería.

\section{INTRODUÇÃO}

O consumo de substâncias psicoativas (SPA) é um fenômeno complexo, permeado por fatores de ordem individual, social, cultural e econômico, sendo muitas vezes incentivado e outras condenado. A condenação está relacionada ao tipo e, a forma de uso, padrão de consumo, local e pessoa que usa. De um modo geral, o consumo de SPA social e de abuso, lícitas e ilícitas, geram estereótipos e preconceitos, sobretudo quando realizado por mulheres. Socialmente, o consumo de substâncias ilícitas apresenta-se incompatível com os papéis e funções de mãe, esposa e cuidadora do lar historicamente atribuídos às mulheres (CRUZ VD, et al.,2014).

Dados do I e II Levantamento Domiciliar sobre o Uso de Drogas Psicotrópicas realizado no Brasil, mostram crescimento do consumo de álcool e de outras substâncias psicoativas entre as mulheres, sobretudo, para aquelas com idade entre 18 e 34 anos (BRASIL, 2003; BRASIL, 2007). O álcool (60,6\%) foi a droga mais utilizada, seguida pelo tabaco $(39,2 \%)$, os benzodiazepínicos $(6,9 \%)$ a maconha $(5,1 \%)$, os orexígenos $(5,1 \%)$ e os anorexígenos (4,5\%) (BRASIL,2007).

$\mathrm{O}$ acesso às drogas na comunidade onde vivem, entre outros aspectos, como o gênero, vínculos precários de trabalho, baixa escolaridade, a influência de amigos e parentes, são apontados como os principais motivos para o início do consumo de SPA entre as mulheres. Paralelo a isso, o envolvimento do parceiro com o comércio ilegal e/ou tráfico contribui para o envolvimento da mulher em tais atividades (MARANGONI SR e OLIVEIRA MLF, 2013). A participação no tráfico de drogas é a principal causa do aprisionamento de mulheres no Brasil. Dados do Departamento Penitenciário Nacional (DEPEN) mostram que 64\% das penas de mulheres estão associadas a condenações por crimes relacionados ao envolvimento com drogas (BRASIL, 2016).

O consumo de drogas é um fator que tornam contexto social e individual da mulher vulnerável dificultando a experiência da maternidade (MARANGONI SR e OLIVEIRA MLF, 2012). Estudo realizado em Maringá com 394 gestantes identificou que aproximadamente 18\% das entrevistadas fizeram uso de drogas de abuso durante a gestação, sendo o consumo mais prevalente para o tabaco $(9,14 \%)$ e para o álcool $(6,09 \%)$ (KASSADA DS, et al., 2013).

Diante das considerações apresentadas e de pesquisas sobre a temática considera-se que o envolvimento com as drogas não se limita ao seu consumo e/ou participação no narcotráfico (envolvimento direto), diz respeito também à convivência com pessoas que adotam tais condutas (envolvimento indireto) (PORTO PN, et al., 2015). Esse envolvimento quer seja direto e/ou indireto, pode ocasionar repercussões sociais e de saúde para as diferentes pessoas envolvidas.

REAS/EJCH |Vol. 11(12) | e795 | DOI: https://doi.org/10.25248/reas.e795.2019 Página 2 de 8 
Mulheres envolvidas com drogas estão mais vulneráveis, sobretudo no contexto de maternidade, que englobam não só o âmbito obstétrico e perinatal, mas também a experiência da maternagem, que consiste na relação afetiva entre a mãe e o bebê. Nesse sentido, este trabalho tem por objetivo: estimar a prevalência e verificar fatores associados ao envolvimento de gestantes com álcool e outras drogas.

\section{MÉTODOS}

Trata-se de um estudo transversal desenvolvido com 268 gestantes atendidas no programa de pré-natal de uma maternidade pública de Salvador-BA. Adotou-se como critérios de inclusão: ser atendida no programa de pré-natal da unidade e apresentar capacidade cognitiva de interagir com a pesquisadora. Como critério de exclusão: idade inferior a 18 anos. A produção do material empírico ocorreu por meio de aplicação de um formulário original, elaborado pela equipe de pesquisa, com 75 questões estruturadas.

Mediante falta de registro específico na unidade acerca da quantidade de gestantes cadastradas, optouse por uma amostra não probabilística e de conveniência. O poder deste estudo foi estimado para a hipótese com prevalência média de $90 \%$ de gestantes envolvidas com álcool e outras drogas, adotando-se um erro de $5 \%$ e nível de significância de 5\% para o cálculo. Para tais dados, encontrou-se um poder de teste de $81,87 \%$.

Os dados foram organizados e processados no software estatístico Statistical Package of Social Science ${ }^{\circledR}$ (SPSS) versão 20.0. Para análise descritiva dos dados utilizou-se a distribuição de frequências. Para a análise bivariada utilizou-se o teste Exato de Fisher e a odds ratio com intervalo de confiança a 95\%. Os preceitos éticos emanados da Resolução 466/12 do Conselho Nacional de Saúde que regulamenta as práticas em pesquisas com seres humanos foram respeitados (BRASIL, 2012). O projeto foi apreciado e aprovado pelo Comitê de Ética da Escola de Enfermagem da Universidade Federal da Bahia, parecer nํ268646.

\section{RESULTADOS}

Do total, $81,4 \%$ relataram ter feito ou estar em uso de algum tipo de droga e conviver com pessoas usuárias e/ou traficantes, caracterizando o duplo envolvimento (Figura 1).

Figura 1 - Prevalência de gestantes envolvidas com álcool e outras drogas atendidas em uma maternidade pública. Salvador, BA, Brasil, 2013.

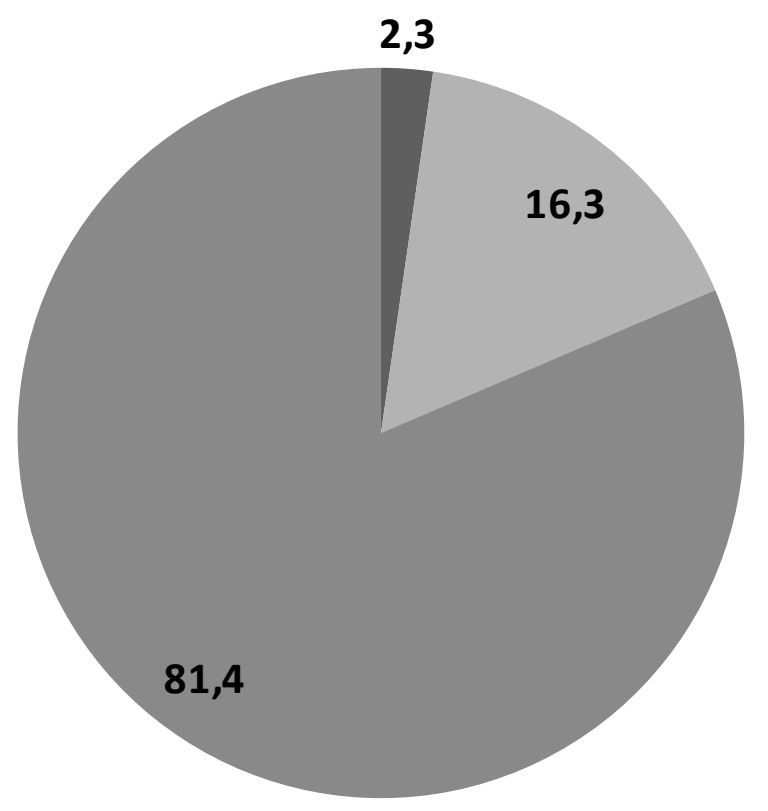

Envolvimento direto

Envolvimento indireto

Envolvimento direto e indireto

Fonte: Próprio autores, 2019. 
A idade média das participantes foi de 26,6 anos (desvio padrão=5,93), com predominância na faixa etária entre 20 e 29 anos (57,8\%). No que se refere à variável raça/cor, 247 (92,2\%) se autodeclararam negras. Quando investigado o grau de escolaridade das participantes, identificou-se que 189 (70,7\%) possuíam entre 9 e 12 anos de estudo, correspondentes às séries do ensino médio. Considerando a ocupação das entrevistadas no momento da pesquisa, 108 (42,9\%) estavam desempregadas ou exerciam atividades não remuneradas, $117(47,2 \%)$ viviam com renda familiar de um a três salários mínimos, $102(38,8 \%)$ eram totalmente dependentes financeiramente do companheiro, ex-companheiro ou familiar.

A prevalência do envolvimento de gestantes com álcool e outras drogas foi de $98,1 \%$. Destas, $2,3 \%$ referiram fazer uso de SPA e não conviver com pessoas usuárias, 16,3\% afirmaram não fazer uso, mas conviver com pessoas usuárias e/ou participantes do narcotráfico, sobretudo pai e/ou companheiro. Dentre as participantes, houve predomínio de consumo de álcool $(84,4 \%)$, seguido pelo tabaco $(14,4 \%)$ e pela maconha $(1,2 \%)$. No que tange aos familiares, destaca-se o consumo de álcool $(77,6 \%)$, tabaco $(31,0 \%)$, maconha $(22,8 \%)$, cocaína $(14,6 \%)$ e crack $(8,9 \%)$. Dentre os familiares envolvidos com álcool e outras drogas destacaram-se o pai $(13,7 \%)$, o companheiro $(13,5 \%)$ e a mãe $(12,4 \%)$.

$\mathrm{Na}$ tabela 1 são apresentados os fatores associados ao uso de SPA pelas entrevistadas. O consumo foi mais prevalente entre as gestantes com idade entre 20 e 29 anos $(47,0 \%)$. A relação entre escolaridade e uso de SPA evidenciou diferença estatisticamente significante $(p$-valor $=0,017)$ entre os grupos.

Tabela 1- Distribuição do uso de substâncias psicoativas em algum momento na vida, de acordo com as variáveis sociodemográficas de gestantes atendidas em uma maternidade pública de Salvador, BA, 2013.

\begin{tabular}{|c|c|c|c|c|c|}
\hline \multirow{2}{*}{ Variáveis } & \multicolumn{2}{|c|}{ Uso de SPAS na vida } & \multirow{2}{*}{${ }^{*} \mathrm{p}$-valor } & \multirow{2}{*}{$\begin{array}{l}\text { Odds } \\
\text { Ratio }\end{array}$} & \multirow[b]{2}{*}{ IC $(95 \%)$} \\
\hline & Não & Sim & & & \\
\hline \multicolumn{6}{|l|}{ Faixa etária } \\
\hline$<20$ anos & $3(1,1 \%)$ & $28(10,5 \%)$ & 0,498 & & \\
\hline $20-29$ anos & $29(10,8 \%)$ & $126(47,0 \%)$ & & 0,60 & $(0,18-2,00)$ \\
\hline$>30$ anos & $16(6,0 \%)$ & $66(24,6 \%)$ & & 0,65 & $(0,18-2,29)$ \\
\hline \multicolumn{6}{|l|}{ Raça } \\
\hline Negra & $44(16,4 \%)$ & $203(75,8 \%)$ & 0,020 & 1,86 & $(0,35-3,38)$ \\
\hline Outras & $4(1,4 \%)$ & $17(6,4 \%)$ & & & \\
\hline \multicolumn{6}{|l|}{ Escolaridade } \\
\hline Analfabeta/fundamental incompleto & $6(2,2 \%)$ & $34(12,7 \%)$ & 0,017 & 2,59 & $(0,72-9,30)$ \\
\hline Fund. Completo/ médio incompleto & $5(1,9 \%)$ & $62(23,1 \%)$ & & 0,61 & $(0,24-1,57)$ \\
\hline Médio completo/superior & $37(13,8 \%)$ & $124(46,3 \%)$ & & & \\
\hline \multicolumn{6}{|l|}{ Religião } \\
\hline Católica & $14(5,2 \%)$ & $81(30,2 \%)$ & 0,01 & 1,10 & $(0,21-56,2)$ \\
\hline Evangélica & $25(9,3 \%)$ & $52(19,4 \%)$ & & 0.89 & $(0,17-45,6)$ \\
\hline Outras & $0(0,0 \%)$ & $10(3,8 \%)$ & & 1,21 & $(0,00-6,44)$ \\
\hline Não possui & $9(3,4 \%)$ & $77(28,7 \%)$ & & & \\
\hline \multicolumn{6}{|l|}{ Renda familiar a } \\
\hline$<1$ salário & $15(6,0 \%)$ & $58(23,4 \%)$ & 0,644 & & \\
\hline 1 a 3 salários & $18(7,3 \%)$ & $99(40,0 \%)$ & & 1,42 & $(0,67-3,01)$ \\
\hline > 3 salários & $10(4,0 \%)$ & $48(19,3 \%)$ & & 1,22 & $(0,51-2,92)$ \\
\hline \multicolumn{6}{|l|}{ Condições de moradia } \\
\hline Casa própria & $41(15,3 \%)$ & $147(54,9 \%)$ & 0,014 & & \\
\hline Alugada & $7(2,6 \%)$ & $73(27,2 \%)$ & & 2,82 & $(1,16-6,83)$ \\
\hline \multicolumn{6}{|l|}{ Acesso ao serviço de saúde } \\
\hline Exclusivamente público & $107(39,9 \%)$ & $113(42,1 \%)$ & 0,56 & 1,00 & \\
\hline Público e privado & $31(11,6 \%)$ & $17(6,4 \%)$ & & 0,62 & $(0,26-1,43)$ \\
\hline
\end{tabular}

Fonte: Próprio autores, 2019. * Valor de p obtido pelo Teste Exato de Fisher $a=248 \quad \alpha=5 \%$. 
A razão de chances de uso de drogas foi 2,6 vezes maior para as mulheres não alfabetizadas ou com ensino fundamental incompleto (OR: 2,59 IC: 0,72 - 9,30). Foi observada associação estatisticamente significante ( $p$-valor $=0,001$ ) entre o uso de SPA e a religião das participantes. O consumo foi mais frequente entre as mulheres católicas $(30,2 \%)$, seguido pelas mulheres que declararam não possuir religião $(28,7 \%)$. Houve predomínio entre as gestantes que faziam uso exclusivamente dos serviços públicos de saúde (82,1\%) e que possuíam renda de um a três salários mínimos (40,0\%). Ao relacionar o uso de SPA e condições de moradia, observou-se diferença estatisticamente significante $(0,014)$. Mulheres que viviam em casa alugada apresentaram 2,8 vezes mais chance de consumir SPA (OR: 2,82 IC: 1,16-6,83) (Tabela 1).

\section{DISCUSSÃO}

No presente estudo, no que se refere às variáveis sociodemográficas da amostra, considerando o envolvimento de SPA em algum momento da vida, houve uma alta prevalência de gestantes envolvidas. Sendo predominantemente o duplo envolvimento, onde as participantes relataram que além de consumir alguma substância, conviviam com pessoas que faziam uso. Não houve relatos sobre participação das gestantes no narcotráfico. Entretanto, algumas mulheres informaram a participação de ex-companheiro e familiares mais distantes, no tráfico de drogas. Não assumir tal conduta para si ou para membros familiares próximos pode estar atrelado às implicações sociais que tal atitude traria, como por exemplo, a discriminação em seu contexto social bem como o possível conflito com a justiça.

Dentre as substâncias consumidas pelas gestantes houve predomínio do álcool, tabaco e maconha. Estudo realizado no Brasil com 8.589 pessoas observou que 60,6\% das mulheres já haviam feito uso do álcool em algum momento na vida e que 36,3\% já havia usado tabaco (GALDURÓZ JCF, et al., 2005).O uso de SPA por mulheres se intersecciona com as questões de gênero, visto que as repercussões do uso de drogas não são as mesmas para homens e mulheres. Mulheres que fazem uso problemático de drogas são mais vulneráveis e estigmatizadas, mais acometidas por problemas de saúde mental, por violências e abusos, se comparadas aos homens (UNODC, 2017). Contudo, o uso de drogas por mulheres continua sendo visto, predominantemente sob a ótica masculina, o que culmina por invisibilizar as mulheres nesse contexto e pôlas como alvo de intervenções tardias com dupla estigmatização, pelo fato de ser mulher e usuária (ALVES TM e ROSA LCS, 2016).

$\mathrm{Na}$ gestação, o uso de substâncias pode desencadear alguns agravos para os bebês, dentre eles: alterações no desenvolvimento do sistema nervoso central, baixo ganho de peso gestacional, malformação fetal, Síndrome Alcoólica Fetal, dentre outras .Apesar desses agravos, muitas mulheres têm dificuldade para diminuir ou interromper o uso (DONALD KA, et al., 2016; FREITAS SR, et al., 2014). A causa da não interrupção pode estar relacionada à dependência da substância, à falta de informação sobre os riscos físicos e psicossociais para o filho, ou ainda, à experiência com gravidez anterior em que não houve complicações para o feto (KASSADA DS, et al., 2014). Independente do contexto destaca-se a importância da equipe de saúde em intervir precocemente em situações de uso/abuso de drogas durante a gestação, bem como a presença de uma rede de apoio à gestante.

As intervenções da equipe de saúde devem estar baseadas na estratégia de redução de danos e minimização de riscos, pautadas nas possibilidades e necessidades individuais. A equipe poderá identificar o padrão de uso (que pode ser problemático ou não), os riscos relacionados ao uso, além de fornecer informações à usuária do serviço, de modo a subsidiar suas escolhas e proporcionar a adesão na construção do projeto terapêutico singular (PTS).

Nesse contexto, destaca-se o protagonismo da enfermeira, tendo em vista ser essa profissional referência para o pré-natal. Considerando que em sua prática, a enfermeira mantém contato direto com a gestante e seus familiares, tem a possibilidade de identificar e intervir em situações que envolvem o uso de drogas. Destarte, o estabelecimento de uma relação pautada na/pela horizontalidade, sem a emissão de juízos de valor, possa contribuir para a potencialidade do cenário assistencial, fundamental para que a gestante se sinta acolhida considerando as suas singularidades e especificidades. No que concerne à faixa etária, observou- 
se que as gestantes com idade igual ou superior a 20 anos apresentaram fator de proteção para o consumo de SPA. Isso pode estar relacionado ao fato de o início do consumo acontecer geralmente durante a adolescência pela inserção em um novo contexto social e influência de amigos, familiares e do ambiente (DOMINGUES, et al., 2014).

Essa fase da vida pode se configurar como período de experimentação das drogas. A(o)s adolescentes poderão apresentar diferentes padrões de uso, tais como: experimentação, uso recreativo, ou ainda uso abusivo/problemático. As implicações desses padrões de uso se correlacionam com fatores intrínsecos, sociais e culturais, reverberando em condições que potencializam a vulnerabilidade.

O estudo apresentou associação estatisticamente significante entre a raça e o consumo de substâncias. A chance de consumo foi maior entre as mulheres negras, comparadas às que se declararam de outras raças. O fato de ser mulher, negra e usar drogas amplia a vivência de situações de vulnerabilidades, fazendo com que elas tenham menos alcance a recursos, como escolaridade, emprego, e acesso aos serviços de saúde. Ademais, experiências de preconceito racial, julgamento que se configuram em discriminação e violências potencializam as vulnerabilidades relacionadas ao uso de substâncias entre as mulheres negras. Destaca-se que os maiores índices de violência geralmente são observados entre as mulheres negras casadas, o que realça a desigualdade de gênero e raça como fatores que aumentam a vulnerabilidade dessas mulheres (GOMES, et al., 2012).

Observou-se associação positiva entre o uso de SPA e a escolaridade das entrevistadas. As mulheres não alfabetizadas/ensino fundamental incompleto apresentaram 2,6 vezes mais chance de se envolverem e/ou consumirem drogas. A baixa escolaridade pode funcionar como um intermediário para outras formas de desvantagem social ao longo da vida, aspectos que podem desencadear situações estressoras e comprometer o enfrentamento de situações de uso/abuso de drogas (CARLINER H, et al., 2017). Por conseguinte, o consumo de drogas pode contribuir para o abandono escolar, sendo que a frequência regular à escola é considerada como fator de proteção para o uso SPA (DOMINGUES S, et al., 2016).

Níveis de escolaridade mais altos podem fornecer à mulher condições materiais (renda, rede de sociabilidade) para enfrentar situações de discriminação, bem como o acesso a informações que desencorajem o consumo de drogas. $O$ status socioeconômico elevado e o acesso à educação estão associados a capacidade autorregular comportamentos de saúde. Dessa forma, mulheres com escolaridade e nível socioeconômico mais elevado podem ter mais recursos para reduzir ou interromper o uso de drogas durante a gestação (CARLINER H, et al., 2017).

Observou-se associação entre a religião e o uso de SPA pelas gestantes. Ser evangélica figurou como fator de proteção para o uso de drogas (PORTO PN e REIS HFT, 2013). Estudos apontam que quanto maior a religiosidade, menor o envolvimento com estas substâncias. Isso pode estar atrelado ao fato de que a religião propicia uma vivência em comunidade que pode funcionar como uma rede de apoio social. Outrossim, a experiência da dimensão transcendental associada aos valores morais que norteiam o comportamento no âmbito religioso favorecem o desenvolvimento de competências para o enfrentamento dos problemas relacionados ao uso de droga.

Identificou-se associação estatística entre o uso de álcool e outras drogas pelas gestantes e a sua condição de moradia. A chance de consumo de SPA entre mulheres foi maior entre as que residiam em casas alugadas. A condição de moradia é um dos fatores que interferem na qualidade de vida dos indivíduos. Entretanto, esse achado necessita ser mais bem explorado, pois a exclusão social implica em privação no acesso a bens e serviços comuns, como por exemplo, a moradia própria, condição tida como sinônima de estabilidade. Nesse sentido, possuir um local adequado para morar, poderá contribuir para o enfrentamento de situações problemas como o risco do uso abusivo de drogas, bem como a redução da exclusão social. Ademais, o uso de álcool e outras drogas estão relacionados a situações de violência doméstica, vínculos familiares fragilizados, rebaixamento da estima, empobrecimento, afastamento social, perda do lar, favorecendo a vivência em situação de rua (MARANGONI SR e OLIVEIRA MLF, 2013; MACÊDO VC, et al., 2017).

REAS/EJCH |Vol. 11(12) | e795 | DOI: https://doi.org/10.25248/reas.e795.2019 Página 6 de 8 
Quanto à renda familiar, houve uma maior proporção de mulheres que consumiam drogas e que possuíam renda familiar de um a três salários mínimos. Associado ao cenário atual determinado pela exclusão e marginalização das populações, o consumo de álcool e outras drogas pode suscitar situações de instabilidade familiar aumentando a vivência de situações de vulnerabilidade (LOPES GT, et al., 2014). Em relação ao uso de SPA pelos familiares das gestantes, houve destaque para o álcool e o tabaco, com registros também do uso de maconha, cocaína e crack, feito predominantemente pelo pai, mãe e companheiro. Esses dados confirmam a presença da problemática das drogas no ambiente familiar das entrevistadas. $O$ fato de o álcool e o tabaco serem drogas legalizadas torna o seu uso aceito socialmente e naturalizado, dessa forma é mais difícil identificar padrões de uso abusivo ou problemático. Nos casos de dependência, torna-se um desafio a diminuição ou interrupção do uso, devido ao fácil acesso à substância.

O uso de drogas ilícitas por familiares contribui para a ampliação da vulnerabilidade, pelo fato do consumo dessas substâncias ainda ser considerada como ato infracional no nosso país, podendo repercutir em implicações com a justiça. Ademais, pessoas que fazem uso de drogas ilícitas frequentemente se colocam em situação de risco para adquirir e consumir tais substâncias. A família exerce papel fundamental no desenvolvimento dos seus membros. O uso problemático de drogas, principalmente o álcool pode exercer influência para a presença de padrão de transmissão transgeracional da dependência. Os sistemas familiares acabam por direcionar a forma de pensar, agir e interagir em sociedade, através de sua estrutura, padrões de comportamento, laços emocionais e histórias compartilhadas (LOPES GT, et al., 2014).

Em uma relação na qual o companheiro mantém determinado padrão de consumo, esse mesmo padrão geralmente é mantido pela mulher, e em alguns casos pode evoluir para o envolvimento com o tráfico de drogas e, consequente conflito com a justiça (MARANGONI SR e OLIVEIRA MLF, 2013).

Além disso, o consumo bem como a comercialização de drogas por familiares pode gerar situações de conflito, medo, estresse e violência para as mulheres, sobretudo quando estão grávidas.

Para além do exposto, vale ressaltar a importância do profissional de Enfermagem no acolhimento e atenção à saúde de mulheres envolvidas com as drogas. No entanto, o que se observa é que a enfermeira encontra dificuldade para lidar com a população usuária de drogas, tanto no que se refere ao relacionamento interpessoal quanto à estrutura dos serviços (VARELA DSS, et al., 2015).

Neste contexto, torna-se imprescindível uma reestruturação dos serviços de saúde e uma formação continuada dos profissionais de saúde para intervir com as questões relacionadas ao envolvimento, no intuito de minimizar os riscos e as vulnerabilidades da população.

\section{CONCLUSÃO}

O presente estudo evidenciou elevada prevalência de gestantes envolvidas com drogas. Esse envolvimento constitui-se como elemento potencial de vulnerabilidade, quer seja pelo consumo de substâncias quer seja pela convivência com pessoas que usam drogas. Portanto, torna-se imprescindível conhecer as diferentes formas de envolvimento e suas repercussões para o contexto de vida dessas mulheres. Nesse sentido, o estabelecimento de estratégias de intervenção precoce em situações de uso/abuso de drogas entre gestantes, pautadas em sua singularidade, considerando o seu contexto de vida, pode minimizar tanto os danos à gestante quanto ao feto.Vislumbra-se com a realização desse estudo, a utilização dos seus resultados na proposição de ações de prevenção ao uso de SPA por gestantes, com ações intersetoriais e compartilhadas com os diversos serviços de atendimento à mulher, o que pode resultar em resultados positivos para ao binômio mãe-filho.

\section{AGRADECIMENTOS E FINANCIAMENTO}

À Maternidade que aceitou e colaborou para o desenvolvimento da pesquisa. Às gestantes que aceitaram participar e enriqueceram o estudo. Ao CNPq que proporcionou a realização da pesquisa através dos editais: MCTI/CNPq/SPM-PR/MDA, Gênero 32/2012 e MCTI/CNPq № 14/2013 - Universal 14/2013. 


\section{REFERÊNCIAS}

1. ALVES TM, ROSA LCS. Usos de substâncias psicoativas por mulheres: a importância de uma perspectiva de gênero. Estudos Feministas. 2016; 24(292): 443-462.

2. BRASIL Ministério da Saúde. Resolução n. 466/12, de 12 de dezembro de 2012. Aprova as diretrizes e normas regulamentadoras de pesquisas envolvendo seres humanos. Brasília, DF; 2012.

3. BRASIL Ministério da Justiça. Departamento Penitenciário Nacional - DEPEN. Levantamento Nacional de Informações Penitenciárias. Brasília, DF; 2016.

4. BRASIL Secretaria Nacional Antidrogas, Gabinete de Segurança Institucional- Presidência da República. I Levantamento domiciliar sobre o uso de drogas psicotrópicas no Brasil. São Paulo, SP; 2003.

5. BRASIL Secretaria Nacional Antidrogas, Gabinete de Segurança Institucional- Presidência da República. II Levantamento domiciliar sobre o uso de drogas psicotrópicas no Brasil: estudo envolvendo as 108 maiores cidades do país. São Paulo, SP; 2007.

6. CARLINER $\mathrm{H}$, et al. Gender Discrimination, educational atteintment and ilict drug use among U.S among. Soc Psychiatry Psychiatr Epidemiol. 2017; 52:279-289.

7. CRUZ VD, et al. Vivências de mulheres que consomem crack. Rev da Rede de Enferm do Nordeste. 2014; 15(4): 639-49.

8. DOMINGUES S, et al. Comportamentos de risco dos adolescentes portugueses e influência do meio ambiente. Nascer e Crescer. 2014; 23(3): 124-133.

9. DONALD KA, et al. Alcohol exposure in utero is associated with decreased gray matter volume in neonates. Metabolic brain disease. 2016; 31(1): 81-91.

10. GOMES N, et al. Homens e mulheres em vivência de violência conjugal: características socioeconômicas. Rev. Gaúcha Enferm. 2012; 33(2): 109-116.

11. FREITAS SR, et al. Tabagismo e gestação: análise de uma amostra de conveniência de puérperas do Hospital Universitário de Santa Maria. Rev AMRIGS. 2014; 58(3): 198-202.

12. GALDURÒZ JCF, et al. Uso de drogas psicotrópicas no Brasil: pesquisa domiciliar envolvendo as 107 maiores cidades do país - 2001. Rev latinamericana enferm. 2005; 13: 888-95.

13. KASSADA DS, et al. Perceptions and practices of pregnant women attended in primary care using illicit drugs. Esc Anna Nery - Rev Enferm. 2014;18(3):428-34.

14. KASSADA DS, et al. Prevalência do uso de drogas de abuso por gestantes. Acta paul enferm. 2013; 26(5): $467-71$.

15. LOPES GT, et al. Percepções de adolescentes sobre uso/dependência de drogas: o teatro como estratégia pedagógica. Esc. Anna Nery. 2014; 18(2): 202-208.

16. MACÊDO VC, et al. 2017. Risk factors for syphilis in women: case-control study. Rev. Saúde Pública vol.51, São Paulo. 2017, Epub Aug 17.

17. MARAGONI SR, OLIVEIRA MLF. Fatores desencadeantes do uso de drogas de abuso em mulheres. Text Context enferm. 2013; 22(3): 662-70.

18. MARAGONI SR, OLIVEIRA MLF. Uso de crack por multípara em vulnerabilidade social: história de vida. Cienc Cuid Saude. 2012; 11(1): 166-72.

19. PORTO PN, REIS HFT. Religiosidade e saúde mental: um estudo de revisão integrativa. Rev baiana saúde pública. 2013; 37(2): 375-93.

20. PORTO PN, et al. Acesso aos serviços de saúde: fatores associados ao envolvimento de gestantes com drogas. Revista Baiana de Enfermagem. 2015; 29(4): 350-60.

21. UNODC Reserch. World Drug Report 2016. 2017;

22. VARELA DSS, et al. Dificuldades de enfermeiros no trabalho com usuários de álcool e outras drogas: revisão integrativa. Rev enferm UFPE on line. 2015; 9(10): 9576-83. 